choice as the most likely one.

The physical, physiological, and pharmacological properties of smooth muscle, and the possible relevance of gastrointestinal hormones and other biogenic substances are discussed in some detail. The significance of mucosal potential, the mechanisms of symptom production, embryological aspects, and oesophageal symptoms associated with other gastrointestinal disease are briefly discussed, and our knowledge about vomiting, belching, aerophagy, oesophageal rupture, and oesophageal abnormalities in various systemic medical disorders is reviewed.

This is an interesting and learned book, erudite yet pleasant and comfortable to read, well produced, and adequately illustrated with diagrams, radiographs, photomicrographs, and references.

D. A. W. EDWARDS

Ileostomy: Surgery, Physiology, and Management By Graham L. Hill (pp. 187; illustrated; price not stated). Grune and Stratton: New York. 1976. This monograph deals comprehensively with all aspects of ileostomy care. The chapters cover historical aspects, operative care, ileostomy care, normal ileostomy physiology, pathophysiology with special reference to diarrhoea metabolism and mechanical problems, rehabilitation, and also the continent ileostomy. It is written by a surgeon who has devoted a tremendous amount of work to this field. It is a most practical and important publication and should be available wherever ileostomies are performed.

Abdominal Ultrasound By Hans Henrik Holm, Jørgen Kvist Kristensen, Sten Nørby Rasmussen, Jan Fog Pedersen, and Søren Hancke (pp. 181; illustrated; D.kr. 200). Munksgaard: Copenhagen. 1976. This comprehensive monograph covers an increasingly important field of advanced diagnosis. The gastrointestinal tract, the biliary system, and pancreas are very well covered with all basic aspects of the subject together with other systems too. The illustrations and reproductions are first class with excellent normal anatomical diagrams for reference in relation to the many scans. It comes from a department where more than 15000 abdominal examinations have been carried out.

Clinics in Gastroenterology Vol. 5: Disorders of Oesophageal Motility. Edited by Michael Atkinson. (Pp. 207; illustrated; subscription rate $£ 13.50$ p.a.). This volume of this popular series is devoted to growing points in basic and clinical studies of the oesophagus. Although this organ has been a popular one for recent books, nevertheless this is, as always, a most up-to-date review written by those most active in this field. It has indeed maintained the high standard of this international series.

Intestinal Ion transport Edited by J. W. L. Robinson. (Pp. 430; illustrated; $£ 12 \cdot 50$ ). MTP: Lancaster, Lancs. 1976. This book provides an up-to-date review of ion and water transport in the intestine in health and disease. It also contains reviews by nephrologists in areas where the ion pumping processes in the kidney are better known than their counterparts in the intestinal tract. It is based on the fourth International Falk Symposium. The main sessions covered are (1) basic mechanisms of ion transport, (2) modifications in intestinal ion transport, (3) adaptation of intestinal ion transport in comparative studies, and (4) transport in the human intestine.

Aspects of Clinical Gastroenterology : Baillière's Medical Transparencies Edited by Oliver James and Ian A. D. Bouchier (24 slides; $£ 8 \cdot 50$ ). This wallet-size publication provides 24 medical transparencies for teaching covering common gastroenterological conditions. Baillière Tindall: London. 1976.

Institut National de la Santé et de la Recherche Médicale This annual report lists the research centres in France supported by the Institut National de la Santé et de la Recherche Medicale (INSERM). Further information and details of research grants may be obtained from Nicole Glynne, D.Sc., Attaché Scientifique de L'INSERM, French Embassy, 41 Parkside, London, SW1X 71P.

\section{Care of Stoma Patients}

This booklet, published by Abbott Laboratories Limited as a service to medicine, reports a day conference held at St. Bartholomew's Hospital, London. It concentrates on all the practical problems of stoma care and is particularly well illustrated. It covers the total care, bringing in the role of the nurse and the patient as well as the medical staff.
CoeliacSociety Newsletter No. 16 provides further valuable information about convenience foods which do or do not contain gluten. For those going on holiday there are lists of cooperative hotels in the United Kingdom, and also travel hints for those going overseas. Some medicines contain gluten and these include Dimotane LA, Dimotapp LA, Donnatal LA, Fybranta, Nardil tablets, Natirose, Nulacin, Saroten tablets, and Veracolate tablets. The address of this excellent society is P.O. Box 181, London NW2 2QY.

\section{Received}

Beecham Foods Nutrition Information Centre Bulletin Number 119 Nutrition and Drugs Part 1. Nutrition Information Centre, Beecham Foods, 11, Stoke Poges Lane, Slough, Berks. 1976.

Authors who wish to have their manuscripts acknowledged are asked to enclose a stamped addressed postcard or an international reply coupon. 